\title{
Pioglitazone prevents cholesterol gallstone formation through the regulation of cholesterol homeostasis in guinea pigs with a lithogenic diet
}

Tao Han ${ }^{1 \dagger}$, Yangge $\mathrm{Lv}^{2+}$, Shijia Wang ${ }^{3}$, Tao $\mathrm{Hu}^{3}, \mathrm{Hao} \mathrm{Hong}{ }^{2}$ and Zan $\mathrm{Fu}^{3 *}$

\begin{abstract}
Background: The cholesterol gallstones diseases (CGD) is highly correlated with metabolic syndrome and type 2 diabetes. The present study aimed to investigate preventive effects of pioglitazone (PIO), an antidiabetic drug, on the CGD in guinea pigs fed with a lithogenic diet (LD).

Methods: The guinea pigs were fed with the LD for 8 weeks. All guinea pigs were grouped as follows: low fat diet; LD; LD plus PIO (4 mg/kg); LD plus PIO (8 mg/kg); LD plus ezetimibe (EZE) $(2 \mathrm{mg} / \mathrm{kg})$. Gallbladder stones were observed using microscopy. The profile of biliary composition, and blood glucose, insulin and lipid were analyzed. The liver or ileum was harvested for determinations of hydroxyl-methyl-glutaryl-CoA reductase (HMGCR), sterol regulatory element-binding proteins 2 (SREBP2), 7a-hydroxylase (CYP7A1), adenosine triphosphate-binding cassette (ABC) sterol transporters G5 and G8 (ABCG5, ABCG8), bile salt export pump (BSEP), Niemann-Pick C1-Like 1 (NPC1L1) and acetyl-coenzyme A cholesterol acyltransferase (ACAT2) by Western blot. The gallbladders were used for histological examination.

Results: The LD successfully induced gallstone. Both pioglitazone and ezetimibe prevented gallstone formation, as well as hepatic and cholecystic damages. Pioglitazone significantly decreased HMGCR and SREBP2, but increased CYP7A1, ABCG5, ABCG8, and BSEP in the liver. Pioglitazone also remarkably decreased NPC1L1 and ACAT2, while increased ABCG5/8 in the intestine. The beneficial alterations of cholesterol and bile acids in the bile, as well as profile of glucose, insulin and lipid in the blood were found in the guinea pigs treated with pioglitazone.
\end{abstract}

Conclusion: Pioglitazone has a noticeable benefit towards the CGD, which is involved in changes of synthesis, transformation, absorption, and transportation of cholesterol.

Keywords: Pioglitazone, Gallstone, Lithogenic diet, Cholesterol, Bile acid

\section{Introduction}

Cholesterol gallstone disease (CGD) is one of the most prevalent and costly diseases. Aging, high calorie diet, hyperinsulinism and metabolic syndrome are among the preeminent risk factors for developing CGD [1]. Major pathogenetic mechanisms are involved in hepatic hypersecretion of cholesterol [2], gallbladder motility disturbances

\footnotetext{
* Correspondence: fu_zan@qq.com

${ }^{\dagger}$ Tao Han and Yangge Lv contributed equally to this work.

${ }^{3}$ Department of General Surgery, the First Affiliated Hospital of Nanjing

Medical University, Nanjing 210000, Jiangsu, China

Full list of author information is available at the end of the article
}

[3], gallbladder inflammation [4], and increased intestinal absorption of cholesterol [2], and altered gut microbiota [5]. Precipitation of excess cholesterol in bile as solid crystals is a prerequisite for cholesterol gallstone formation. Novel approaches against these pathogenetic factors are urgently needed to be developed for the prevention and treatment of the CGD.

Dietary cholesterol is trafficked into enterocyte by Niemann-Pick C1-like 1 (NPC1L1) protein [6], and can be reversely transported to the intestinal lumen by adenosine triphosphate-binding cassette $(\mathrm{ABC})$ sterol transporters G5 and G8 (ABCG5, ABCG8) [7, 8]. After cholesterol

(C) The Author(s). 2019 Open Access This article is distributed under the terms of the Creative Commons Attribution 4.0 International License (http://creativecommons.org/licenses/by/4.0/), which permits unrestricted use, distribution, and 
passes through the enterocyte membrane, it is esterified by acetyl-coenzyme A cholesterol acyltransferase 2 (ACAT2), and subsequently transported in the chylomicrons circulating then reaches the liver for biliary secretion $[9,10]$. HMG-CoA reductase (HMGCR) and $7 \alpha-$ hydroxylase (CYP7A1) play important roles in maintaining cholesterol homeostasis. HMGCR is a rate-limiting enzyme in hepatic cholesterol biosynthesis while CYP7A1 is a rate-limiting enzyme transforming cholesterol to bile acid (BA). The ABCG5/8 effluxes sterols including cholesterol from hepatocytes and enterocytes into the bile and intestine, respectively, and may therefore simultaneously increase biliary cholesterol excretion and lower plasma cholesterol levels [8]. The bile salt export pump (BSEP), also known as ABCB11, is the major canalicular BA transporter [11]. Cholesterol gallstone formation is associated with downregulation of BSEP expression on the canalicular membrane of hepatocytes [12]. In addition, cholesterol metabolism is tightly controlled by some feedback mechanisms, including regulation of the sterol regulatory element-binding protein 2 (SREBP2) and liver X receptor $\alpha$ (LXR $\alpha)$ [13-15].

Pioglitazone, a thiazoledinedione (TZD), is a highly selective peroxisome proliferator-activated receptor- $\gamma$ (PPAR $\gamma$ ) agonist. Widely used as antidiabetic agent, pioglitazone not only has an impact on glycemic control but also has been shown to increase HDL and decrease LDL and TG in diabetes [16]. All anti-diabetic drugs have varying effects on lipid profile but overall pioglitazone has shown more favorable lipid lowering effect compared to other antidiabetics including rosiglitazone $[17,18]$. In more recent years, the focus on pioglitazone has been intensified due to its novel biological roles. Herein, we investigated effects of pioglitazone on cholesterol gallstone formation as well as NPC1L1, ACAT2, HMGCR, CYP7A1, ABCG5/8, and BSEP in the guinea pigs with a lithogenic diet.

\section{Materials and methods Materials}

Pioglitazone (PIO) was purchased from MSD Pharma (Singapore) Pte. Ltd. Ezetimibe (EZE) was purchased from DEYUAN PHARM Co. Ltd. (Shanghai, China). The antibodies of SREBP-2(sc-13,552), CYP7A1 (sc-293, 193), FXR (sc-25,309), LXR (sc-271,064), BSEP (sc-74, 500), CCK-AR(sc-514,303) and NPC1L1(sc-166,802) were purchased from Santa Cruz Biotechnology Co. Ltd. (Santa Cruz, CA). The antibodies of HMGCR (ab174830), ABCG5 (ab87116) and ABCG8 (ab126493) were from Abcam Technology Co. Ltd. (Cambridge, UK). The antibodies of ACAT2 (13294S) and PPARY (2443S) were from Cell Signaling Technology Inc. (Boston, USA). Histone H3, $\beta$-actin and the secondary antibodies were from Bioworld Technology Co. Ltd.
(Minneapolis, MN, USA). BCA assay kit was from Beyotime (Nanjing China). Amplex Red cholesterol $(\mathrm{CH})$ assay kit was from the Invitrogen (USA). All other chemical or Biological reagents were commercially available.

\section{Animals}

Male guinea pigs $(350 \pm 20 \mathrm{~g})$ were obtained from the Yangzhou University Medical Center (Yangzhou, China) and were housed under the environmentally controlled conditions of 12-h light-dark cycles in a full-barrier facility with a relative humidity of $60 \pm 5 \%$ and temperature of $22 \pm 2{ }^{\circ} \mathrm{C}$. The guinea pigs had free access to water and food. All diets were prepared and pelleted by Yangzhou University Medical Center. The lithogenic diet (LD) refers to chow diet supplemented with approximately $1.25 \%$ cholesterol, $0.5 \%$ bile salt and $15 \%$ fat as well as $0.0033 \%$ or $0.0067 \%$ pioglitazone or $0.0017 \%$ ezetimibe. After 1 week of adaptation, the guinea pigs were randomly divided into 5 groups and treated as follows: low fat diet (CTRL), LD, LD plus PIO $4 \mathrm{mg} / \mathrm{kg}$ (PIO-L), LD plus PIO $8 \mathrm{mg} / \mathrm{kg}$ (PIO-H), LD plus EZE $2 \mathrm{mg} / \mathrm{kg}$. The guinea pigs were fed with the LD containing pioglitazone or ezetimibe for 8 weeks. The experiments were performed according to the National Institutes of Health Guide for the Care and Use of Laboratory Animals (NIH publication No. 86-23, revised 1996), and approved by the guidelines of the Institutional Animal Care and Use Committee of China Pharmaceutical University.

\section{Biochemical assays}

The levels of blood glucose, triglyceride (TG), lowdensity lipoprotein $\mathrm{CH}$ (LDL-C) and high-density lipoprotein $\mathrm{CH}$ (HDL-C) were measured according to the instructions of their assay kits (Nanjing Jiancheng Bioengineering Institute, Nanjing, China). The serum insulin was measured using the insulin ELISA kit (Millipore, USA). The $\mathrm{CH}, \mathrm{BA}$, phospholipid (PL) in the serum or bile were measured using the respective kits. Furthermore, the $\mathrm{CH}$ saturation index (CSI) was calculated using the following equation: actual molar percentage of $\mathrm{CH}$ in bile/the largest soluble $\mathrm{CH}$ concentration at a given bile molarity in the Carey table [19].

\section{Histological evaluation of the liver and gallbladder}

The liver and gallbladder specimens were prepared and fixed in $4 \%$ neutral-buffered formaldehyde overnight. The specimens were embedded in paraffin, cut into 4$\mu \mathrm{m}$-thick slices, stained with haematoxylin and eosin $(\mathrm{H}$ \& E). After dewaxing, staining, dehydration, transparency and sealing, the specimens of liver and gallbladder were examined under a light microscope. 


\section{Western blot analysis}

Liver and ileum were chopped into small pieces and homogenized in $0.5 \mathrm{ml}$ of lysis buffer (phenylmethylsulfonyl fluoride (PMSF): RIPA =1:9). The dissolved proteins were collected from the supernatant after centrifugation at $12,000 \times \mathrm{g}$ for $15 \mathrm{~min}$. Protein concentration was determined by BCA assay kit (Beyotime, Nanjing China). The protein was separated on $12 \%$ SDS-polyacrylamide gel and transferred onto the PVDF membrane. After blocking the nonspecific site with blocking solution ( $5 \%$ nonfat dry milk), the membrane was incubated overnight with specific primary antibody at $4 \mathrm{C}$ with primary antibodies: HMGCR (1:300), SREBP-2 (1:500), CYP7A1 (1:500), ABCG5 (1:600), ABCG8 (1:300), LXR $\alpha$ (1:500), $\operatorname{BSEP}(1: 500), \quad$ NPC1L1(1:500) and ACAT2(1:1000). Histone H3 (1:1000) and $\beta$-actin (1:3000) served as an internal control, respectively. Subsequently, the membrane was washed with TBST followed by incubation with appropriate horseradish peroxidase-conjugated secondary antibody (1:5000) at the room temperature for $1 \mathrm{~h}$. The blots were again washed with TBST and then developed with the ECL Plus Western Blotting Detection System (Tanon Science \& Technology Co. Ltd.)

\section{Statistical analyses}

The data were expressed as means \pm SD All data, unless specified, were analyzed by a one-way ANOVA followed by a Dunnett's post hoc analysis for multiple comparisons. All analyses were carried out using SPSS, version 20.0. A probability value $(P)$ of $<0.05$ was considered statistically significant.

\section{Results}

Pioglitazone treatment inhibits cholesterol gallstone formation in the Guinea pigs fed with LD

In our study, the CTRL group had a cholesterol gallstone formation rate of $9.1 \%$, the LD group showed a cholesterol gallstone formation rate of $85.7 \%$, a statistically significant increase over the rate of the CTRL group. The gallstone formation rate in the PIO-L and PIO-H groups were 53.8 and $38.5 \%$, respectively. The gallstone formation rate was $25.0 \%$ in the guinea pigs treated with a hypolipidemic agent, ezetimibe, as positive control. Thus, both of pioglitazone and ezetimibe reduced the rates of gallstone formation in the guinea pigs. The Fig. 1c shows photomicrographs of the bile, liquid crystals, and gallstone samples. The bile of control guinea pigs did not contain any crystals or sludge, while the bile of the LD-fed guinea pigs contained sludge particles and crystals. The PIO-L, PIO-H or EZE group contained few crystals and sludge in their bile samples.
Pioglitazone treatment alters biliary composition and CSI in the Guinea pigs fed with LD

As shown in Table 1, the levels of both $\mathrm{CH}$ and $\mathrm{BA}$ were significantly increased in gallbladder bile of the guinea pigs in the LD group $(* * 0.01)$, compared with those in the CTRL group. The $\mathrm{CH}$ level significantly declined and BA level further increased in the gallbladder bile after treatment with high-dose pioglitazone or ezetimibe $\left({ }^{* *} P<0.01\right)$. Treatment with lowdose pioglitazone also changed levels of biliary $\mathrm{CH}$ and BA, but there were not statistical significances. In addition, treatment with low- or high-dose pioglitazone and ezetimibe significantly inhibited the LDinduced increase in CSI in the guinea pigs.

\section{Effects of pioglitazone on metabolic parameters}

As shown in Table 2, compared with CTRL group, treatment with the LD had no significant effects on blood glucose and insulin, while treatment with high-dose pioglitazone significantly decreased levels of blood glucose and insulin (blood glucose: ${ }^{* * P} P<0.01$; insulin: ${ }^{*} P<0.05$ ). The levels of $\mathrm{CH}, \mathrm{LDL}-\mathrm{C}$ and TG increased and HDL-C level decreased in the peripheral blood of the guinea pigs after feeding LD for 8 weeks $(* * P<0.01)$. Treatment with high-dose pioglitazone or ezetimibe significantly decreased CH, LDL-C and TG levels, and increased HDL$\mathrm{C}$ level in the serum of guinea pigs $(\mathrm{CH}: * * P<0.01$; LDL-C: $* P<0.05$; TG: $* P<0.01$; HDL-C: $* P<0.05)$. Treatment with low-dose pioglitazone showed some tendency of regulation in blood glucose, insulin and lipid profile, but did not reach statistical significance $(P>$ 0.05).

Pioglitazone treatment prevents the histological damages of liver or gallbladders in the Guinea pigs fed with LD

The structure of the normal liver was as follows: hepatic plates radiate from the central vein, and the plates are separated at irregular intervals by sinusoids without abnormal morphological changes. Some characteristic histology features of the CGD were observed in the livers of the guinea pigs fed with LD for 8 weeks, including steatosis, spotty necrosis, hepatocyte ballooning, and lobular inflammation. Compared with the LD group, the extents of hepatocellular steatosis, necrosis and hepatocyte ballooning were markedly decreased in the PIO-H and EZE groups. These results indicated that both ezetimibe and pioglitazone treatment produced protective effects on the liver damage induced by the LD (Fig. 2a). Gallbladder inflammation is indicated by thickened gallbladder wall, infiltrated inflammatory cells in the stromal layer, and submucosal vasodilatation in the CGD. All these signs were seen in the LD group, but few inflammatory signs were observed in the PIO-H and EZE groups (Fig. 2b). 
A

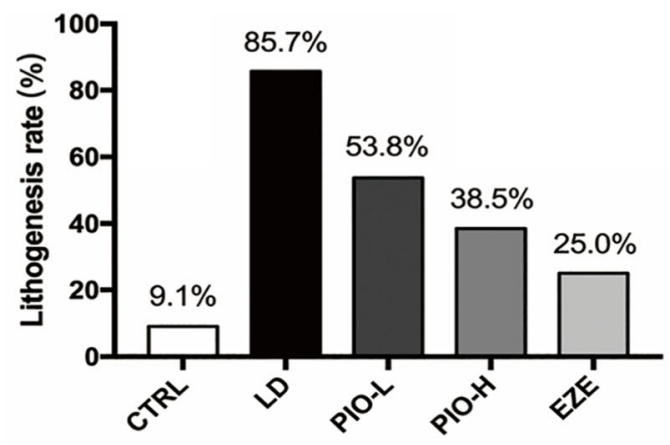

B

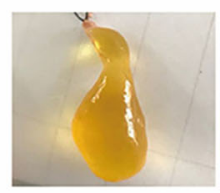

CTRL

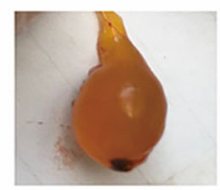

LD

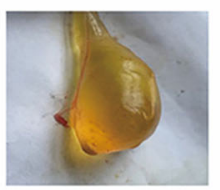

PIO-L

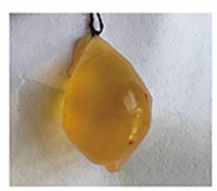

PIO-H

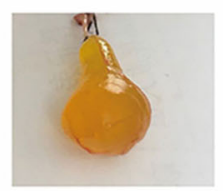

EZE

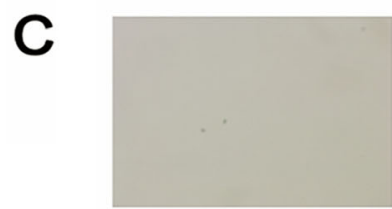

CTRL

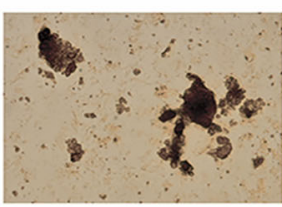

LD

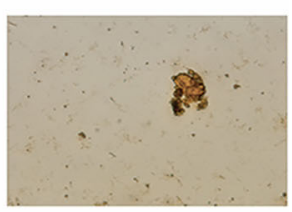

PIO-L

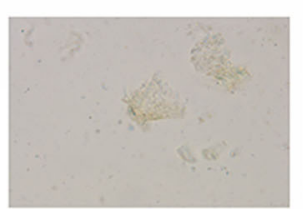

PIO-H

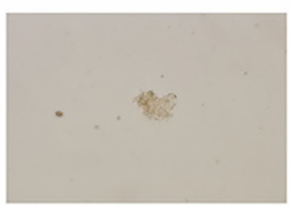

EZE

Fig. 1 Pioglitazone treatment inhibits cholesterol gallstone formation in the guinea pigs fed with LD. a lithogensis rate; b macroscopic appearances of the gallbladders; $\mathbf{c}$ photomicrographs of bile, liquid crystals and gallstones

Table 1 Pioglitazone treatment alters biliary composition and CSI in the guinea pigs fed with LD

\begin{tabular}{llllll}
\hline Variables & CTRL & LD & PIO-L & PIO-H & EZE \\
\hline CH (mmol/L) & $2.93 \pm 1.14^{* *}$ & $8.39 \pm 1.64$ & $7.05 \pm 1.68$ & $5.65 \pm 1.33^{* *}$ & $5.07 \pm 1.30^{* *}$ \\
BA (mmol/L) & $56.09 \pm 9.86^{* *}$ & $77.02 \pm 9.86$ & $80.59 \pm 9.77$ & $92.26 \pm 8.46^{* *}$ & $85.47 \pm 8.91$ \\
PL (mmol/L) & $22.89 \pm 5.15$ & $25.65 \pm 4.89$ & $26.33 \pm 4.68$ & $27.47 \pm 5.27$ & $26.22 \pm 4.56$ \\
CSI & $0.57 \pm 0.17^{* *}$ & $1.13 \pm 0.15$ & $0.85 \pm 0.13^{*}$ & $0.70 \pm 0.13^{* *}$ & $0.67 \pm 0.10^{* *}$ \\
\hline
\end{tabular}

Values are expressed as means \pm SD $(n=8) .{ }^{*} P<0.05$ and ${ }^{* *} P<0.01$ versus $L D$ group 
Table 2 Effects of pioglitazone on metabolic parameters

\begin{tabular}{llllll}
\hline Variables & CTRL & LD & PIO-L & PIO-H & EZE \\
\hline Body weight (g) & $479.39 \pm 50.97$ & $511.83 \pm 70.00$ & $490.13 \pm 40.78$ & $435.46 \pm 69.62^{*}$ & $492.46 \pm 63.29$ \\
Blood glucose (mmol/L) & $3.35 \pm 0.52$ & $3.90 \pm 0.63$ & $3.39 \pm 0.68$ & $2.99 \pm 0.39^{* *}$ & $3.43 \pm 0.51$ \\
Insulin (mmol/L) & $2.45 \pm 0.53$ & $3.00 \pm 0.54$ & $2.58 \pm 0.59$ & $2.40 \pm 0.53^{*}$ & $2.51 \pm 0.29$ \\
CH (mmol/L) & $3.60 \pm 0.74^{* *}$ & $6.28 \pm 0.91$ & $5.85 \pm 0.77$ & $5.04 \pm 1.07^{*}$ & $4.36 \pm 0.81^{* *}$ \\
TG (mmol/L) & $0.37 \pm 0.07^{* *}$ & $0.63 \pm 0.10$ & $0.50 \pm 0.10^{*}$ & $0.44 \pm 0.10^{* *}$ & $0.47 \pm 0.09^{* *}$ \\
LDL-C(mmol/L) & $1.39 \pm 0.19^{* *}$ & $2.02 \pm 0.30$ & $1.84 \pm 0.27$ & $1.72 \pm 0.27^{*}$ & $1.64 \pm 0.18^{*}$ \\
HDL-C (mmol/L) & $1.03 \pm 0.20^{* *}$ & $0.69 \pm 0.19$ & $0.77 \pm 0.14$ & $0.91 \pm 0.10^{*}$ & $0.94 \pm 0.18^{*}$ \\
\hline
\end{tabular}

Values are expressed as means \pm SD $(\mathrm{n}=8) .{ }^{*} P<0.05$ and ${ }^{* * *} P<0.01$ versus LD

Pioglitazone promoted LD-induced decrease in HMGCR or SREBP-2 and inhibited the decrease in CYP7A1 in the liver Western blot was employed to examine the expression of HMGCR or SREBP-2 in the liver of guinea pigs challenged with LD plus different drugs or the LD alone for 8 weeks. The Fig. 3 showed that LD caused significant decreases in HMGCR and SREBP-2 $(* P<0.05)$, and high-dose pioglitazone enhanced the decreases in both proteins in the liver of guinea pigs $\left({ }^{*} P<0.05\right)$. However, ezetimibe didn't produce same effects. In addition, LD induced significant reduction of CYP7A1 expression in the liver $\left.{ }^{* *} P<0.01\right)$, and this effect was reversed by pioglitazone $\left({ }^{*} P<0.05\right)$. Ezetimibe also produced similar effect. These results show that pioglitazone like ezetimibe reduces biosynthesis and transformation of $\mathrm{CH}$ in liver.

\section{Pioglitazone increased the expression of hepatic ABCG5, ABCG8 and BSEP}

As shown in Fig.4, LD didn't change the expression of ABCG5 and ABCG8, but LD plus low- high- dose pioglitazone significantly increased expression of ABCG5 and ABCG8 in the liver (ABCG5:** $P<0.01$; ABCG8: ${ }^{*} P<$ 0.05). Ezetimibe didn't show these effects. Pioglitazone and ezetimibe remarkably inhibited the LD-induced decrease of BSEP in liver (BSEP: ${ }^{*} P<0.05$, ${ }^{* *} P<0.01$ ). These

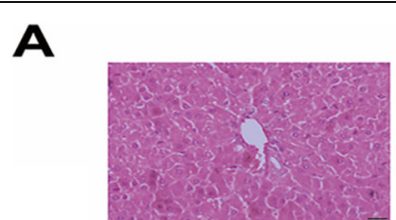

CTRL

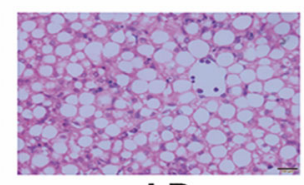

LD

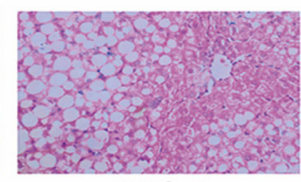

PIO-L

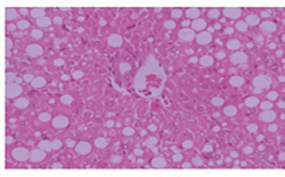

PIO-H

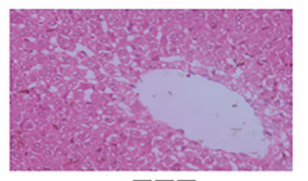

EZE

B

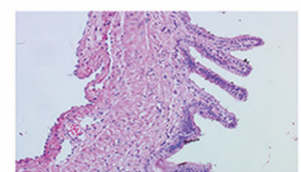

CTRL

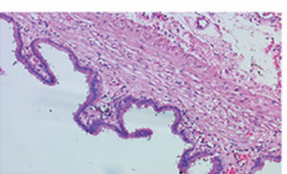

LD

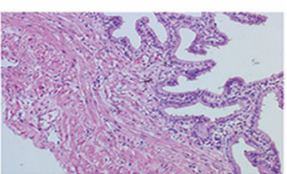

PIO-L

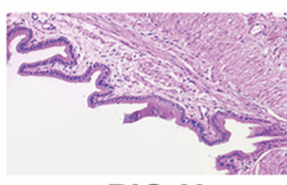

PIO-H

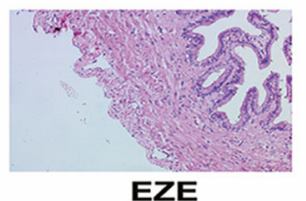

Fig. 2 Pioglitazone treatment prevents the histological damages of liver or gallbladder in the guinea pigs fed with LD. a Histological examination of the liver by H\&E staining. $\mathbf{b}$ Histological examination of the gallbladder by H\&E stainin 


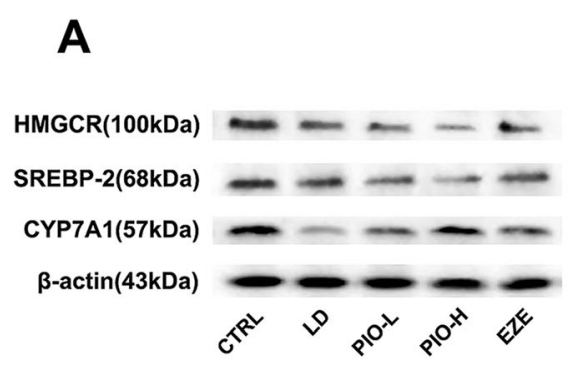

B

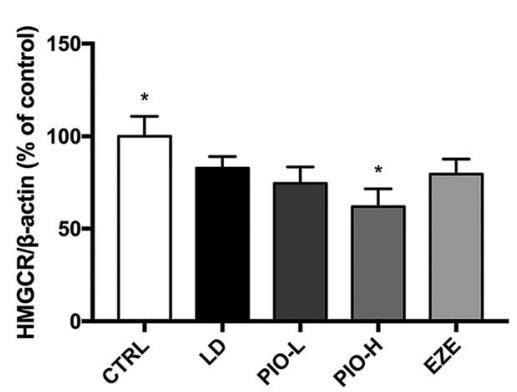

C

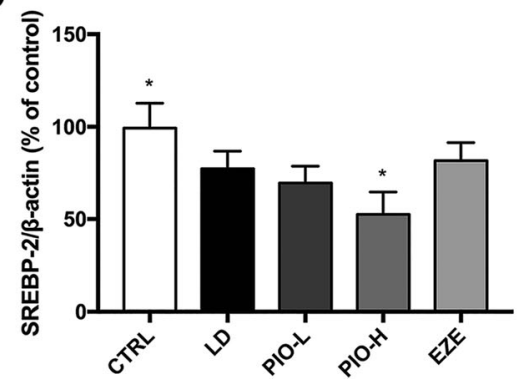

D

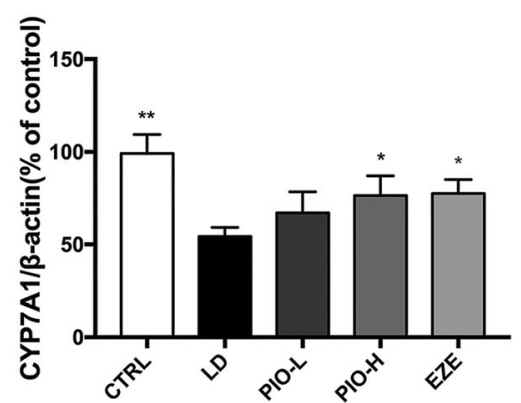

Fig. 3 Pioglitazone promotes LD-induced decrease of HMGCR or SREBP-2 and inhibited decrease in CYP7A1 in liver. a Western blot analysis of HMGCR, SREBP2 and CYP7A1 protein; $\mathbf{b}$ Quantification of HMGCR expressed as the ratio (as a percentage) of control; c Quantification of SREBP-2 expressed as the ratio (as a percentage) of control; $\mathbf{d}$ Quantification of CYP7A1 expressed as the ratio (as a percentage) of control. Values shown are means $\pm \mathrm{SD}, n=4 .{ }^{*} p<0.05$ and ${ }^{* *} p<$ 0.01 versus LD group
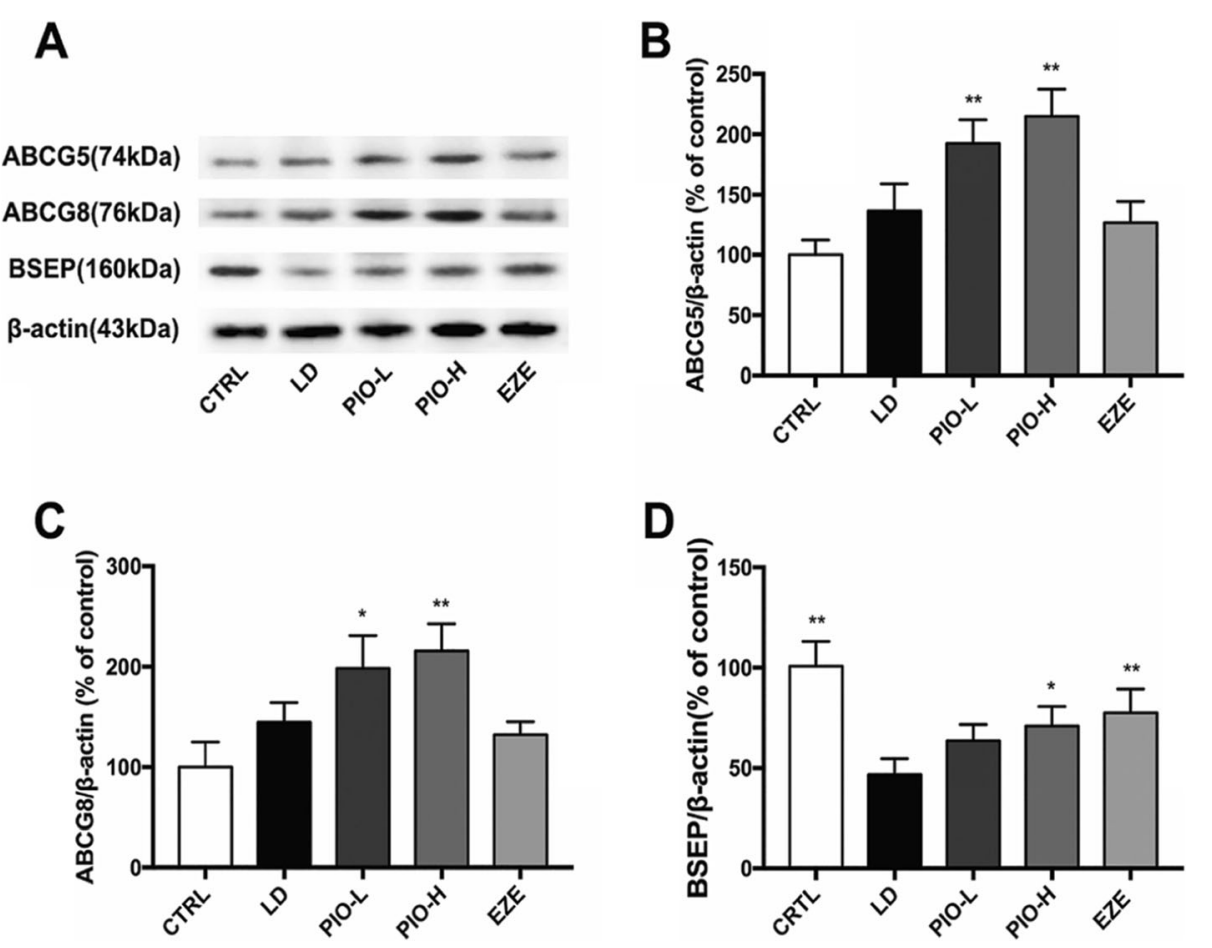

Fig. 4 Pioglitazone increases expression of hepatic ABCG5, ABCG8 and BSEP. a Western blot analysis of ABCG5, ABCG8 and BSEP protein; $\mathbf{b}$ Quantification of ABCG5 expressed as the ratio (as a percentage) of control; c Quantification of ABCG8 expressed as the ratio (as a percentage) of control; $\mathbf{d}$ Quantification of BSEP expressed as the ratio (as a percentage) of control. Values shown are means $\pm S D, n=4 .{ }^{*} p<0.05$ and ${ }^{* *} p<0.01$ versus $L D$ group 
results suggest that pioglitazone could increase the transport of $\mathrm{CH}$ or $\mathrm{BA}$ in liver.

\section{Pioglitazone increased ABCG5 and ABCG8 and decreased NPC1L1 and ACAT2 in the intestine}

As shown in Fig. 5, pioglitazone rather than ezetimibe obviously increased the expression of ABCG5 and ABCG8 (**P $P<0.01)$, and pronouncedly inhibited the LDinduced elevation of NPC1L1 and ACAT2 in the intestine (NPC1L1: ${ }^{* *} P<0.01$; ACAT2: ${ }^{*} P<0.05,{ }^{* *} P<0.01$ ). These results suggest that pioglitazone would reduce cholesterol absorption in intestine.

Pioglitazone changed PPARY, LXRa and FXR in the liver or intestine

As shown in Fig.6, pioglitazone rather than ezetimibe obviously increased expression of PPAR $\gamma$ and LXR $\alpha$ in both liver and intestine (liver: PPAR $\gamma:{ }^{*} P<0.05,{ }^{* *} P<$ 0.01; LXR $\alpha:{ }^{*} P<0.05,{ }^{* *} P<0.01$; intestine: PPARy: ${ }^{*} P<$ 0.05 , $* P<0.01$; LXR $\alpha: * * P<0.01$ ), while pioglitazone and ezetimibe obviously inhibited LD-induced decrease in expression of FXR in the liver $\left({ }^{*} P<0.05\right)$, suggesting that LXR $\alpha$ and FXR might be involved in regulation of the expression of the genes related to cholesterol homeostasis.

\section{Discussion}

The present study clearly indicated that pharmacological treatment with pioglitazone effectively prevented the formation of CGD induced by the LD in guinea pigs, accompanied by decreased $\mathrm{CH}$, increased $\mathrm{BA}$, and lower CSI in the bile as well as lower blood glucose, insulin, TG and $\mathrm{CH}$ levels. Pioglitazone also ameliorated hepatic and gallbladder tissue damage induced by the LD, which

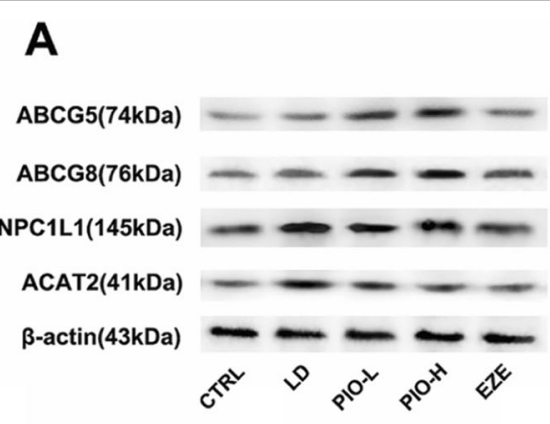

\section{B}

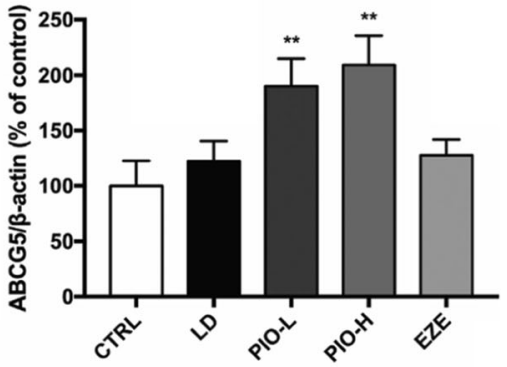

C

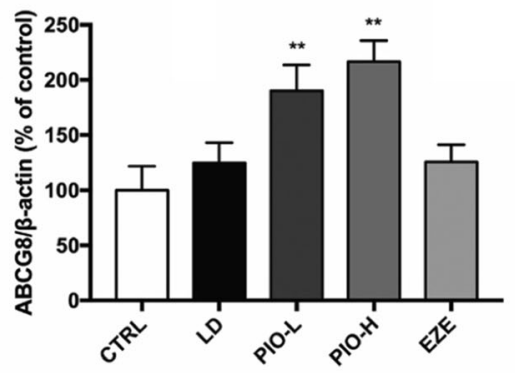

D

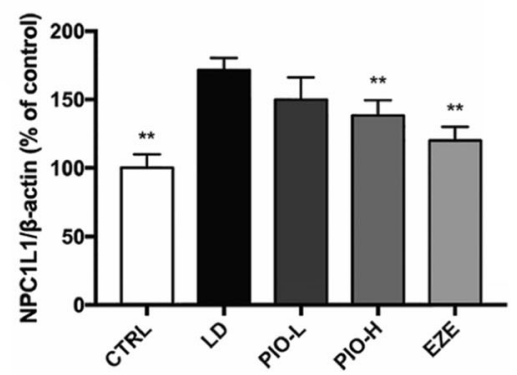

E

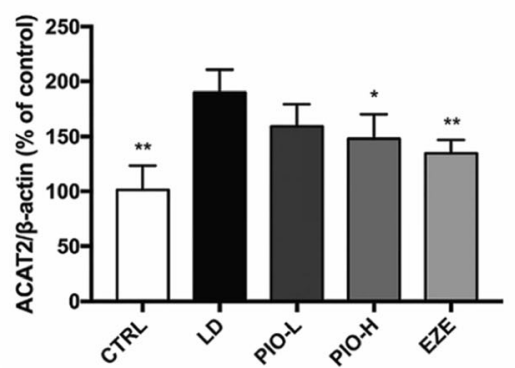

Fig. 5 Pioglitazone increases $A B C G 5$ and $A B C G 8$ and decreases NPC1L1 and ACAT2 in intestine. a Western blot analysis of $A B C G 5, A B C G 8$, NPC1L1 and ACAT2 protein; b Quantification of ABCG5 expressed as the ratio (as a percentage) of control; c Quantification of ABCG8 expressed as the ratio (as a percentage) of control; d Quantification of NPC1L1 expressed as the ratio (as a percentage) of control; e Quantification of ACAT2 expressed as the ratio (as a percentage) of control. Values shown are means $\pm S D, n=4 .{ }^{*} p<0.05$ and ${ }^{* *} p<0.01$ versus LD group 
A

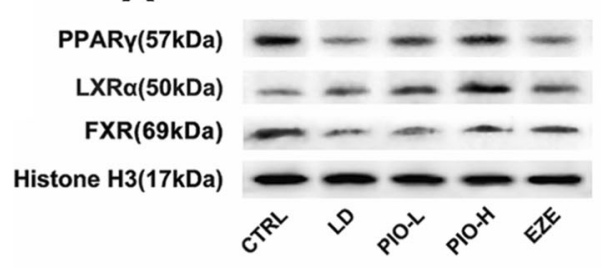

C

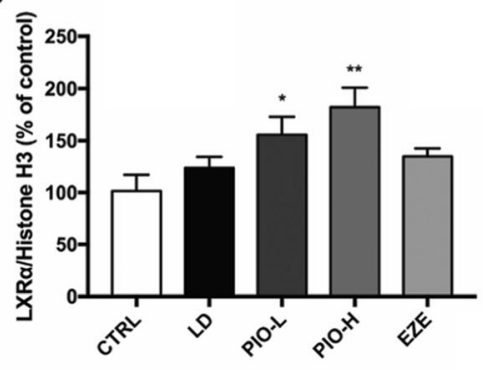

E
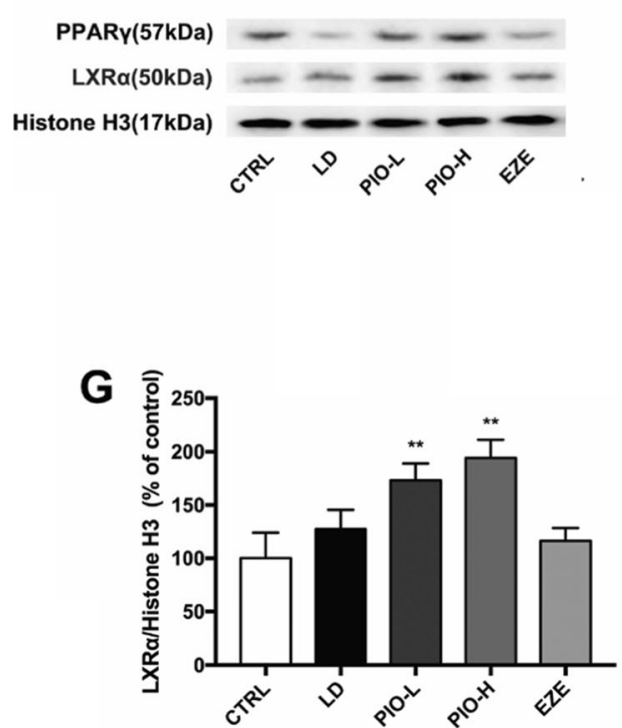

$B \widehat{\overline{\mathrm{\theta}}}$

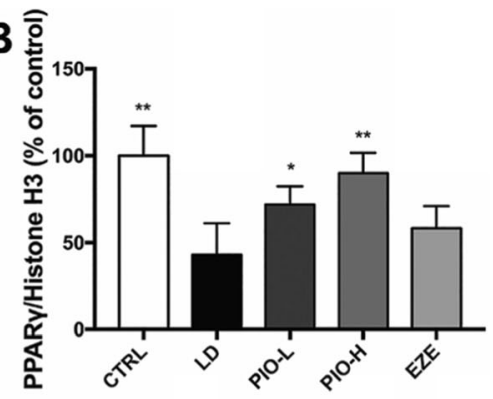

D

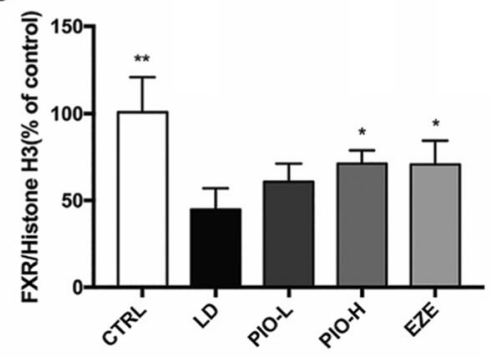

$\mathbf{F}$

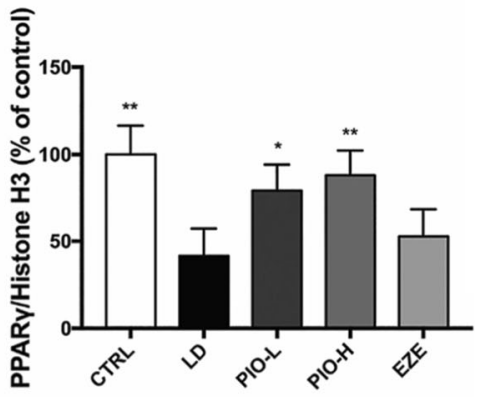

Fig. 6 Pioglitazone changes PPARY, LXRa and FXR in liver or intestine. a Western blot analysis of PPARY, LXRa and FXR protein in liver; $\mathbf{b}$ Quantification of PPARY expressed as the ratio (as a percentage) of control; c Quantification of LXRa expressed as the ratio (as a percentage) of control; $\mathbf{d}$ Quantification of FXR expressed as the ratio (as a percentage) of control; e Western blot analysis of PPARY and LXRa protein in intestine; $\mathbf{f}$ Quantification of PPARY expressed as the ratio (as a percentage) of control; $\mathbf{g}$ Quantification of LXRa expressed as the ratio (as a percentage) of control. Values shown are $m e a n s \pm S D, n=4$. ${ }^{*} p<0.05$ and ${ }^{* *} p<0.01$ versus LD group

could also influence the formation of CGD. The further studies showed that pioglitazone treatment resulted in remarkable decreases in NPC1L1 and ACAT2, and increases in ABCG5/8 in the ileum as well as significant decreases in HMGCR and SREBP2, increases in CYP7A1, ABCG5/8, and BSEP in the liver. All of these effects are contributive to restoration of cholesterols homeostasis and prevention of the CGD.

The formation of CGD refers to three main pathophysiological mechanisms including the supersaturation of bile with cholesterol, gallbladder hypomotility and destabilization of bile by proteins or pathogens. Among 
these, cholesterol supersaturation of bile remains the most critical determinant of the CGD. During the process of the CGD, the homeostasis of cholesterol is highly disrupted, and events involve cholesterol intake, metabolism, and synthesis. It is known that cholesterol derived from the intestine provides the first major source for cholesterol pool and influences biliary cholesterol secretion [20], and that high absorption of cholesterol contributes to gallstone formation through this pathway [21]. Animal studies indicate that high cholesterol absorption efficiency and subsequent rapid biliary secretion of cholesterol promote cholesterol gallstone disease [21]. The LD-induced $\mathrm{CH}$ gallstone models are widely used to elucidate the mechanism of CGD pathogenesis [22, 23]. Herein, administration to guinea pigs for 8 successive weeks disrupted the homeostasis of cholesterol indicated by higher levels of $\mathrm{CH}$ in both the blood and the bile, which results mainly from the increase in cholesterol intake. Our results showed that the LD-fed guinea pigs had a $85.7 \%$ gallstone formation rate. The present data also further confirmed the preventive effects of pioglitazone on the CGD in the model of guinea pigs as shown in the mouse model [24]. As a key protein controlling intestinal cholesterol absorption, NPC1L1 was expressed by enterocytes, identified as a target of ezetimibe [25]. Ezetimibe, a selective NPC1L1 inhibitor, prevents gallstone formation in mice, indicates that NPC1L1 is a valid therapeutic target against the CGD [26]. Free cholesterol absorbed in the intestine through NPC1L1 forms a cholesterol ester catalyzed by ACAT2 and then enters the blood circulation through the lymphatic system, while the unesterified cholesterol is secreted into the intestinal lumen by ABCG5/8, which is the opposite to action of NPC1L1. In the present studies, we found that LD induced significant upregulation of both NPC1L1 and ACAT2 rather than ABCG5/G8 in the ileum, and pioglitazone, like ezetimibe, markedly inhibited expression of NPC1L1 and ACAT2. Importantly, pioglitazone instead of ezetimibe significantly increased intestinal ABCG5/G8 expression. These suggest that pioglitazone might more effective than ezetimibe in reduction of cholesterol intake in intestine.

The hepatocyte is the major site for peripheral uptake and synthesis of cholesterol, and excess cholesterol is directly secreted into bile or converted into bile salts [27]. Endogenous synthesis of cholesterol is regulated at the transcriptional level by the SREBP-2 pathway and is regulated at the post-transcriptional level by the ratelimiting enzyme HMGCR [28]. When the intracellular cholesterol level is high, SREBP-2 forms a complex structure with Scap (SREBP cleavage-activating protein) and Insig-1 (insulin-induced gene-1) through the Cterminal domain, thereby inhibiting SREBP-2 regulation of HMGCR transcription. Our data also showed that expression of HMGCR or SREBP-2 was decreased through feedback regulation in the guinea pigs fed with $\mathrm{LD}$, and pioglitazone instead of ezetimibe make HMGCR and SREBP-2 further deceases in the liver. These are supported by the previous data that PPAR $\gamma$ activation caused reductions of the SREBP-2 and its target genes HMGCR, as well as the reduction of cholesterol synthesis in vitro [29, 30]. Synthesis of bile acids from cholesterol is the dominant pathway for eliminating excess cholesterol in the body. Bile acids may reduce the synthesis of itself through feedback inhibition of CYP7A1, when they are too much [31]. It is known that the expression of CYP7A1 can be regulated by several negative feedback loops, such as FXR-SHP-LRH-1 pathway [32]. It was found that there is a feedback repression of CYP7A1 when the guinea pigs fed with LD, and both of pioglitazone and ezetimibe reversed this inhibition in the present studies. The conversion of cholesterol into bile acids is regulated by LXR $\alpha$-mediated stimulation of CYP7A1 transcription [33]. LXR $\alpha$ is the predominant receptor in the liver, and is directly regulated by PPARY $[34,35]$. Therefore, this might explain the up-regulation of CYP7A1 after PPARy upregulation resulting from the treatment with pioglitazone. BSEP is the major hepatic BA canalicular efflux transporter. Both of pioglitazone and ezetimibe increased gallbladder biliary BA content, which is involved in the enhancement of BSEP-mediated BA transport.

ABCG5 and ABCG8 are target genes of LXRs [7, 36]. LXR $\alpha$ is directly regulated by PPAR $[34]$, and thus this might explain how LXR $\alpha$ cooperates with PPAR $\gamma$ to expedite cholesterol efflux in the intestine and liver. FXR plays a key role in the regulation of BA levels in enterohepatic circulation and has been shown important in preventing gallstone formation in susceptible mice. FXR deficient mice were found to be more susceptible to cholesterol gallstone formation than wild-type mice when fed with a lithogenic diet [37]. Moreover, treatment of C57L mice, which are susceptible to cholesterol gallstone formation, with a potent FXR agonist prevents gallstone formation [37]. FXR activation also reduced hepatic expression of sterol regulatory element binding protein 1c, resulting in reductions of triglyceride and cholesterol content in the liver and amelioration of hyperlipidemia [38]. There is a cross-talk between FXR and PPAR $\gamma$, which is necessary for a proper response to PPAR $\gamma$ activation [39]. Thus, FXR also participate in the improvement of cholesterol homeostasis by pioglitazone.

\section{Conclusions}

In summary, as shown in Fig. 7, as a PPAR $\gamma$ agonist, pioglitazone prevented the LD-induced formation of the CGD, and restored cholesterol homeostasis. These effects are associated with regulating expression of 


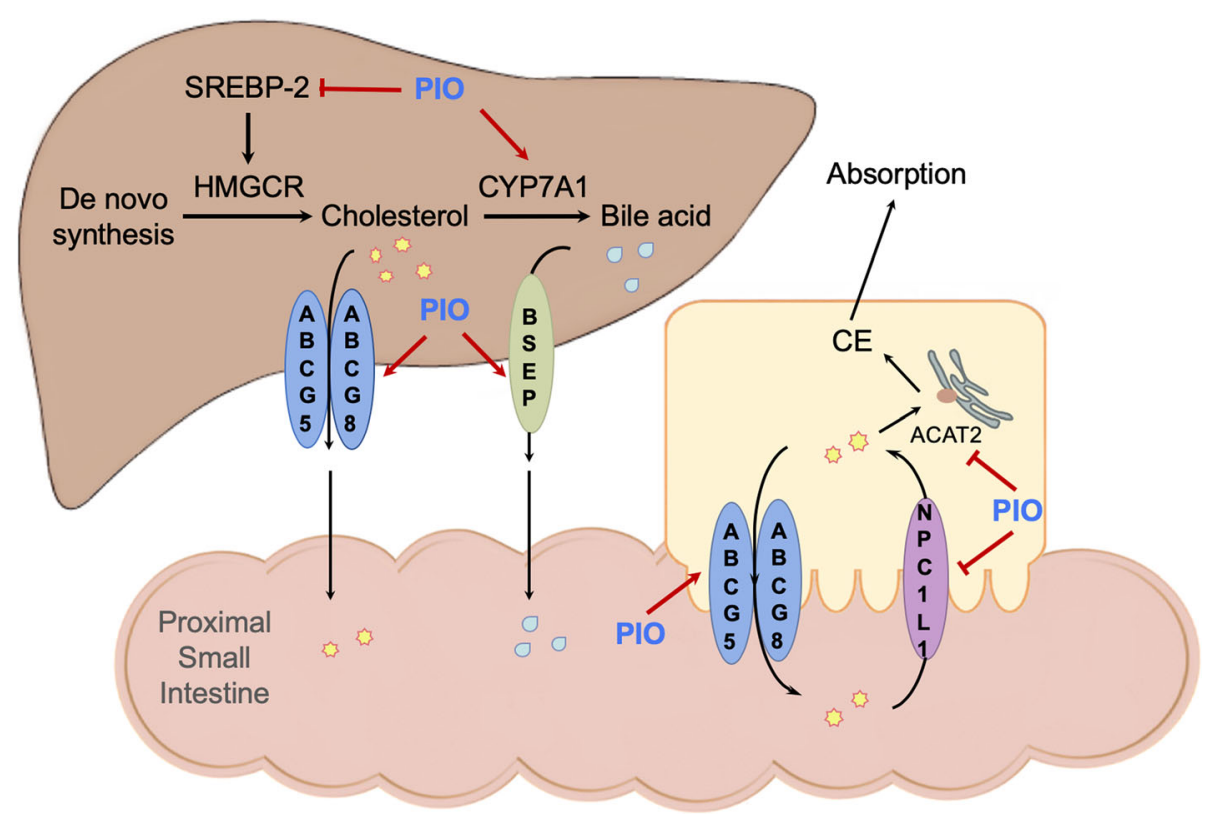

Fig. 7 The illustration shows the mechanism of pioglitazone (PIO) on regulation of cholesterol homeostasis

HMGCR and SREBP2, CYP7A1, ABCG5/8, BSEP, NPC1L1 and ACAT2 in the liver or intestine as well as amelioration of hepatic and gallbladder tissue damage induced by the LD. PPAR $\gamma$, LXR $\alpha$ and FXR participated in regulation of these genes associated with absorption, synthesis, transformation, and transportation of $\mathrm{CH}$ together or independently. We thus suggest that PPAR $\gamma$ agonist pioglitazone might have beneficial effects for the CGD.

\section{Abbreviations \\ ABCG5, ABCG8: Adenosine triphosphate-binding cassette (ABC) sterol trans- porters G5 and G8; ACAT2: Acetyl-Coenzyme A cholesterol acyltransferase; BA: Bile acid; BSEP: Bile salt export pump; CGD: Cholesterol gallstone disease; $\mathrm{CH}$ : Cholesterol; CSI: CH saturation index; CYP7A1: 7a-hydroxylase; EZE: Ezetimibe; HDL-C: High-density lipoprotein CH; HMGCR: Hydroxyl- methyl-glutaryl-CoA reductase; LD: Lithogenous diet; LDL-C: Low-density lipoprotein CH; LXRa: Liver X receptor a; NPC1L1: Niemann-Pick C1-like 1; PIO: Pioglitazone; PL: Phospholipid; PPARY: Peroxisome proliferator-activated receptor-ү; SREBP2: Sterol regulatory element-binding proteins 2; \\ TG: Triglyceride; TZD: Thiazoledinedione}

\section{Acknowledgements}

We sincerely thank Yangge Lv and Xuan Ke for technical assistance, and Yifeng Du for language assistance.

\section{Authors' contributions}

$\mathrm{TH}$ and ZF designed the study; ZF and $\mathrm{HH}$ analyzed all data, and were major contributors in writing the manuscript; $T H$ and $Y L$ performed the histological examination of liver and gallbladder and Western blot; SW and TH performed biochemical assays. All authors read and approved the final manuscript.

\section{Funding}

This work was supported by the funds from the Natural Science Foundation of China (81470881 to Zan Fu).

\section{Availability of data and materials}

All data generated or analyzed during this study are included within the article.

\section{Ethics approval}

All animals used in this study were treated strictly according to the National Institutes of Health Guidelines of the Care and Use of Laboratory Animals. All procedures were approved by the Animal Care Review Committee, China Pharmaceutical University, China.

\section{Consent for publication}

Not applicable.

\section{Competing interests}

The authors declare that they have no competing interests.

\section{Author details}

'Department of Intensive Care Unit, the First Affiliated Hospital of Nanjing Medical University, Nanjing 210000, Jiangsu, China. ${ }^{2}$ Department of Pharmacology, China Pharmaceutical University, Nanjing 210009, China. ${ }^{3}$ Department of General Surgery, the First Affiliated Hospital of Nanjing Medical University, Nanjing 210000, Jiangsu, China.

Received: 7 August 2019 Accepted: 29 November 2019

Published online: 11 December 2019

\section{References}

1. Portincasa P, Moschetta A, Palasciano G. Cholesterol gallstone disease. Lancet. 2006;368:230-9.

2. Portincasa P, Wang DQ. Intestinal absorption, hepatic synthesis, and biliary secretion of cholesterol: where are we for cholesterol gallstone formation? Hepatology. 2012;55(5):1313-6.

3. Lavoie B, Nausch B, Zane EA, et al. Disruption of gallbladder smooth muscle function is an early feature in the development of cholesterol gallstone disease. Neurogastroenterol Motil. 2012;24(7):e313-24.

4. Maurer KJ, Carey MC, Fox JG. Roles of infection, inflammation, and the immune system in cholesterol gallstone formation. Gastroenterology. 2009; 136(2):425-40

5. Fremont-Rahl JJ, Ge Z, Umana C, et al. An analysis of the role of the indigenous microbiota in cholesterol gallstone pathogenesis. PLoS One. 2013;8(7):e70657.

6. Lauridsen BK, Stender S, Frikke-Schmidt R, Nordestgaard BG, TybjærgHansen A. Genetic variation in the cholesterol transporter NPC1L1, ischaemic vascular disease, and gallstone disease. Eur Heart J. 2015;36: 1601-8. 
7. Repa JJ, Berge KE, Chris P, Richardson JA, Helen H, Mangelsdorf DJ. Regulation of ATP-binding cassette sterol transporters ABCG5 and ABCG8 by the liver $X$ receptors alpha and beta. J Biol Chem. 2002;277:18793-800.

8. Stefan S, Ruth F-S, Nordestgaard BG, Tybjærg-Hansen A. The ABCG5/8 cholesterol transporter and myocardial infarction versus gallstone disease. J Am Coll Cardiol. 2014;63(20):2121-8.

9. Grenier E, Garofalo C, Delvin E, Levy E. Modulatory role of PYY in transport and metabolism of cholesterol in intestinal epithelial cells. PLoS One. 2012;7: e40992.

10. Jahangir I, Mohamed B, Rudel LL, M Mahmood H. Intestine-specific MTP and global ACAT2 deficiency lowers acute cholesterol absorption with chylomicrons and HDLs. J Lipid Res. 2014;55:2261-75.

11. Fuchs $C D$, Paumgartner $G$, Wahlström $A$, et al. Metabolic preconditioning protects BSEP/ABCB11-/- mice against cholestatic liver injury. J Hepatol. 2017;66:95-101.

12. Jing K, Bin-Bin L, Shuo-Dong W, Yu W, Qing-Quan J, En-Ling G. Enhancement of interaction of BSEP and HAX-1 on the canalicular membrane of hepatocytes in a mouse model of cholesterol cholelithiasis. Int J Clin Exp Pathol. 2014:7:1644-50.

13. Bengoechea-Alonso MT, Ericsson J. SREBP in signal transduction: cholesterol metabolism and beyond. Curr Opin Cell Biol. 2007:19(2):215-22.

14. Goedeke L, Fernándezhernando $C$. Regulation of cholesterol homeostasis. Cell Mol Life Sci. 2012;69(6):915-30.

15. Zhao C, Dahlmanwright K. Liver $X$ receptor in cholesterol metabolism. J Endocrinol. 2010;204(3):233-40.

16. Spanheimer R, Betteridge DJ, Tan MH, Ferrannini E, Charbonnel B, PROactive Investigators. Long-term lipid effects of pioglitazone by baseline antihyperglycemia medication therapy and statin use from the PROactive experience (PROactive 14). Am J Cardiol. 2009;104:234-9.

17. Betteridge DJ. Effects of pioglitazone on lipid and lipoprotein metabolism. Diabetes Obes Metab. 2007:9:640-7.

18. Inbaraj SD, Josephine IG. Comparative study of lipid and glycemic effects of pioglitazone, rosiglitazone with glibenclamide in patients with type 2 diabetes and dyslipidemia. Global J Pharmacol. 2014;8:107-13.

19. Carey MC. Critical tables for calculating the cholesterol saturation of native bile. J Lip Res. 1978;19:945-55.

20. Wang HH, David Q-HW. Reduced susceptibility to cholesterol gallstone formation in mice that do not produce apolipoprotein B48 in the intestine. Hepatology. 2010;42:894-904.

21. Wang $\mathrm{QH}$, Zhang $\mathrm{L}$, Wang $\mathrm{HH}$. High cholesterol absorption efficiency and rapid biliary secretion of chylomicron remnant cholesterol enhance cholelithogenesis in gallstone-susceptible mice. Biochim Biophys Acta. 1733; 2005:90-9.

22. Ja Kyung K, Soo Min C, So Hee K, et al. N-3 polyunsaturated fatty acid attenuates cholesterol gallstones by suppressing mucin production with a high cholesterol diet in mice. J Gastroenterol Hepatol. 2012;27:1745-51.

23. Song $X Y, X u S, H u J F$, et al. Piperine prevents cholesterol gallstones formation in mice. Eur J Pharmacol. 2015;751:112-7.

24. Wang G, Han T, Wang S, Chen M, Sun Y, Fu Z. Peroxisome proliferatoractivated receptor- $\gamma$ prevents cholesterol gallstone formation in C57bl mice by regulating bile acid synthesis and enterohepatic circulation. Biomed Res Int. 2018;2018:7475626.

25. Dimova LG, de Boer JF, Plantinga J, et al. Inhibiting cholesterol absorption during lactation programs future intestinal absorption of cholesterol in adult mice. Gastroenterology. 2017;153:382-5.

26. Silvia Z, Héctor M, Lorena A, et al. Ezetimibe prevents cholesterol gallstone formation in mice. Liver Int. 2010:28:935-47.

27. Russell DW, Setchell KD. Bile acid biosynthesis. J Biochem. 1992;31:4737-449.

28. Jeon TI, Osborne TF. SREBPs: metabolic integrators in physiology and metabolism. Trends Endocrinol Metab. 2012;23:65-72.

29. Klopotek A, Hirche F, Eder K. PPAR gamma ligand troglitazone lowers cholesterol synthesis in HepG2 and Caco-2 cells via a reduced concentration of nuclear SREBP-2. Exp Biol Med (Maywood). 2006;231:1365-72.

30. Han T, LV Y, Wang S, Hu T, Hong H, Fu Z. PPARY overexpression regulates cholesterol metabolism in human L02 hepatocytes. J Pharmacol Sci. 2019; 139:1-8

31. Shapiro H, Kolodziejczyk AA, Halstuch D, Elinav E. Bile acids in glucose metabolism in health and disease. J Exp Med. 2018;15:383-96.

32. Goodwin $B$, Jones $S A$, Price RR, et al. A regulatory cascade of the nuclear receptors FXR, SHP-1, and LRH-1 represses bile acid biosynthesis. Mol Cell. 2000;6:517-26
33. Chiang JY, Kimmel R, Stroup D. Regulation of cholesterol 7alphahydroxylase gene (CYP7A1) transcription by the liver orphan receptor (LXRalpha). Gene. 2001;262:257.

34. Chawla A, Boisvert WA, Lee $\mathrm{CH}$, et al. A PPAR gamma-LXR-ABCA1 pathway in macrophages is involved in cholesterol efflux and atherogenesis. Mol Cell. 2001;7:161-71.

35. Zhu Y, Li Y. Liver $X$ receptors as potential therapeutic targets in atherosclerosis. Clin Invest Med. 2009;32(5):E383-94.

36. Guorong X, Hai L, Lu-Xing P, et al. FXR-mediated down-regulation of CYP7A1 dominates LXRalpha in long-term cholesterol-fed NZW rabbits. J Lip Res. 2003:44:1956-62.

37. Moschetta A, Bookout AL, Mangelsdorf DJ. Prevention of cholesterol gallstone disease by FXR agonists in a mouse model. Nat Med. 2004;10: 1352-8.

38. Mencarelli A, Renga B, Distrutti E, Fiorucci S. Antiatherosclerotic effect of farnesoid X receptor. Am J Physiol Heart Circ Physiol. 2009;296:H272-8.

39. Mouaadh A, Sandrine C, Christian D, et al. The farnesoid X receptor regulates adipocyte differentiation and function by promoting peroxisome proliferator-activated receptor-gamma and interfering with the Wnt/betacatenin pathways. J Biol Chem. 2010;285:36759-67.

\section{Publisher's Note}

Springer Nature remains neutral with regard to jurisdictional claims in published maps and institutional affiliations.
Ready to submit your research? Choose BMC and benefit from:

- fast, convenient online submission

- thorough peer review by experienced researchers in your field

- rapid publication on acceptance

- support for research data, including large and complex data types

- gold Open Access which fosters wider collaboration and increased citations

- maximum visibility for your research: over $100 \mathrm{M}$ website views per year

At $\mathrm{BMC}$, research is always in progress.

Learn more biomedcentral.com/submissions 